BMJ Open Sport \& Exercise Medicine

\title{
Could Virtual Reality play a role in the rehabilitation after COVID-19 infection?
}

\author{
Merlijn Smits (D, ${ }^{1} \mathrm{~J}$ Bart Staal (D), ${ }^{2,3}$ Harry van Goor (D) 1
}

To cite: Smits M, Staal JB, van Goor H. Could Virtual Reality play a role in the rehabilitation after COVID-19 infection? BMJ Open Sport \& Exercise Medicine 2020;0:e000943. doi:10.1136/ bmjsem-2020-000943

Received 17 August 2020 Revised 25 September 2020 Accepted 9 October 2020
Check for updates

(C) Author(s) (or their employer(s)) 2020. Re-use permitted under CC BY-NC. No commercial re-use. See rights and permissions. Published by BMJ.

'Department of Surgery, Radboud University Medical Center, Nijmegen, The Netherlands

${ }^{2} \mathrm{IQ}$ healthcare, Radboud University Medical Center, Nijmegen, The Netherlands ${ }^{3}$ School for Allied Health, Musculoskeletal Rehabilitation Research Group, HAN University of Applied Sciences, Nijmegen, The Netherlands

\section{Correspondence to}

Merlijn Smits;

Merlijn.smits@radboudumc.nl

\section{ABSTRACT}

Post-COVID-19 patients, particularly those who needed high care, are expected to have high needs for physical, psychological and cognitive rehabilitation. Yet, the resources needed to provide rehabilitation treatment are expected to be inadequate because healthcare systems faced a shortage of high-quality treatment of these symptoms already before the COVID-19 crisis emerged in patients with comparable needs. In this viewpoint, we discuss the potential of Virtual Reality (VR) administering fast, tailor-made rehabilitation at a distance, and offering a solution for the impending surge of demand for rehabilitation after COVID-19. VR consists of a headmounted display (HMD) that can bring the user by computer-generated visuals into an immersive, realistic multi-sensory environment. Several studies on VR show its potential for rehabilitation and suggest VR to be beneficial in post-COVID-19. The immersion of VR may increase therapy adherence and may distract the patient from experienced fatigue and anxiety. Barriers still have to be overcome to easily implement VR in healthcare. We argue that embedding VR in virtual care platforms would assist in overcoming these barriers and would stimulate the spread of VR therapy, both for post-COVID-19 patients in the present and possibly for other patients with similar rehabilitation needs in the future.

\section{INTRODUCTION}

Even as many countries struggle to care for critically ill COVID-19 patients, a new challenge has emerged: Post-Intensive Care Syndrome after coronavirus infection, or as we name it, PICS-COV. ${ }^{1}$ Patients who receive intensive care are known to be at high risk for physical, psychological and cognitive impairments, a constellation known as PICS. A subset of COVID-19 patients who were admitted to the intensive care unit (ICU) frequently required several weeks of such intensive care; furthermore, traditional PICS preventive measures, such as reduction of environmental stressors, early mobility or writing an ICU diary, are virtually impossible due to infection control precautions, prone positioning and deprivation of social contact. ${ }^{2}$ Consequently, COVID-19 patients potentially have a substantial need for physical, psychological and cognitive rehabilitation after discharge from the ICU and the hospital. ${ }^{3}$ In

\section{Key points}

- Post-COVID-19 patients, particularly those who needed high care, are expected to have high needs for physical, psychological and cognitive rehabilitation.

- Virtual Reality provides potential to administer fast, tailor-made rehabilitation at a distance to postCOVID-19 patients.

- Embedding Virtual Reality in virtual care platforms would ease implementing Virtual Reality in healthcare.

the present viewpoint, we discuss how Virtual Reality (VR) can provide rehabilitation to post-COVID-19 patients. We summarise the needs for rehabilitation of patients who suffered from COVID-19, particularly those after ICU admission, the potential of VR rehabilitation and the expected challenges to implement home-based VR rehabilitation.

Several consensus statements and position papers concerning treatment and prevention of PICS-COV have already been put forth by physiotherapists' and behavioural therapists' professional organisations. ${ }^{4-6}$ These are largely comparable with those addressing PICS before the COVID-19 pandemic, although with certain alterations addressing respiratory function recovery and hygiene measures. Nonetheless, the resources needed to successfully implement these healthcare interventions are inadequate because healthcare systems faced a shortage of high-quality PICS treatment even before the COVID-19 crisis emerged. $^{7}$ Traditional PICS treatments also do not account for the additional emotional and societal side-effects of this particular crisis, such as social distancing, limitation of family members' visits and consultations by primary caregivers when patients are discharged. VR provides healthcare practitioners with the means to administer fast, temporary and tailormade rehabilitation services at a distance, and offers a solution to address the impending surge of demand for PICS-COV therapy.

\section{WHAT IS VIRTUAL REALITY?}

In literature, the term Virtual Reality is used for a wide range of technological interventions. Those vary between head-mounted 
displays (HMDs), flat-screen projections and large simulators. All share the ability to bring the user by computergenerated visuals into an immersive multi-sensory environment. At its conception in the late 20th century, VR was expensive, difficult to operate and often led to nausea. Current VR headsets, like the Oculus Go (Facebook, Inc.; Menlo Park, USA), Oculus Quest (Facebook, Inc.; Menlo Park, USA) or HTC Vive (HTC corporation; Taoyuan, Taiwan) have potential to provide safe and affordable rehabilitation at home. Their side-effects are reportedly limited. ${ }^{8}$ These headsets allow VR becoming part of telemedicine: any type of healthcare delivery at a distance, employing technologies like telephone calls, videoconferencing and remote monitoring. ${ }^{10}$ The articles referenced here analyse any type of VR. We refer to their conclusions when the content described could as well be applied in current VR HDMs for home-based rehabilitation.

\section{PICS-COV AND VR}

Recovering COVID-19 patients have deficits that are usually amenable to physiotherapy interventions, for example, resistance training for respiratory muscle weakness, and exercise-based rehabilitation for impaired mobility and other physical functions. ${ }^{11}{ }^{12}$ Similar physiotherapy interventions have been provided by VR. Studies have shown post-stroke virtual rehabilitation improves balance, ability to deal with environmental challenges, and walking speed compared to other interventions. ${ }^{13}$ Reviews have also demonstrated VR's efficacy in increasing joint mobility and motor function for patients with chronic musculoskeletal disorders, positive impacts on rehabilitation of individuals with geriatric syndromes and better balance among children with Cerebral Palsy. ${ }^{8}{ }^{14}$ Although these are different pathologies from COVID-19, the similarities in treatment goals and hurdles (eg, compliance, anxiety for movements and fatigue) suggest that VR is also beneficial in post-COVID-19.

In addition to physical rehabilitation, we should anticipate a need for psychological support to reduce depression, stress, post-traumatic stress disorder (PTSD) and anxiety in COVID-19 patients. An intensive care admission is a fearsome experience, especially when a patient is supported by mechanical ventilation as is common in COVID-19. Depression occurs in approximately $30 \%$ of recovering patients, post-traumatic stress disorder (PTSD) in $10-50 \%$ of patients and anxiety in $70 \%$ of PICS patients. ${ }^{2}$ Psychological therapy targeting these indications can be offered through VR. There are many applications designed to target stress reduction, depression, anxiety and even PTSD. Applications, for example, can provide mindfulness by employing guided meditation in natural settings which might decrease experience of pain and stress. ${ }^{15}$ With regard to depression, there exist several promising applications which provide gamified cognitive behavioural therapies, psychoeducation, behavioural activation and cognitive restructuring. ${ }^{16}$ In addition, VR has demonstrated success in the application of exposure therapy—a modality in which participants are virtually confronted with their fears to decrease anxiety or PTSD. ${ }^{17} 18$

High physical and psychological stressors during ICU admissions routinely result in cognitive disabilities such as memory loss, inability to concentrate, and may confer higher risks of dementia. Without intervention, such impairments can last for years after discharge.${ }^{19}$ Cognitive interventions, including memory exercises and exercises for building optimism, may reduce these symptoms. ${ }^{20}$ These can easily be provided through VR, and allow for exercises to be completed at individualised pacing. ${ }^{21}$

\section{ADVANTAGES OF VIRTUAL REALITY FOR PICS-COV REHABILITATION}

VR rehabilitation as part of telemedicine solutions has several advantages compared to traditional rehabilitation. First, considering our society's continuing need for social distancing, therapy can be given at a distance. Second, VR allows therapists to treat patients simultaneously. While therapists must monitor sessions at a distance in some cases, live support is generally not required, increasing patients' autonomy and reducing the workload of therapists. Other telemedicine solutions, such as video calls and therapy by videos or games on a flat-screen, offer (some) similar advantages. What makes VR particularly different is that it provides its users with a $3 \mathrm{D}$ immersive environment. The immersion makes therapy more fun and reduces distraction from the external environment. Both might increase therapy adherence and engagement. Besides, VR may serve to distract patients from experienced fatigue and anxiety which would otherwise hinder their ability to move. ${ }^{22}$ In addition, VR offers extensive tools compared to other telemedicine services that allow therapists through the use of a dashboard and tracking software to constantly monitor treatment adherence and patients' performance.

\section{BARRIERS TO IMPLEMENTATION}

The COVID-19 crisis has stimulated the adoption of telemedicine technologies in several domains. ${ }^{23}$ This opens the door to apply VR treatment as part of telemedicine. Nonetheless, there are a few challenges that must be addressed before VR rehabilitation can be implemented. First, VR toolkits in the three domains of physical, mental and cognitive therapy will need to be created. Existing VR applications are already abundant. The challenge for VR developers and therapists will be to rapidly select and in some cases (re)design applications that are safe and effective in use for PICSCOV rehabilitation at home, based on patientreported experiences that thus far are still scarce and dispersed. Another barrier to implementation is the safety of using VR HMDs at home. Patients, especially those with imbalance, have a potential safety risk when doing mobility exercises for example, accidental falls. To increase safety, patients should be instructed well on doing mobility exercises in a playground free from objects. Fortunately, new VR HMDs have safety 
precautions, switching from the virtual environment to the real environment when approaching objects. We also recommend doing all psychological and cognitive exercises sitting on a chair. Other concerns relate to costs of purchasing a VR headset and education on VR. Education sessions and technical support are required to take any patient through the use of VR headsets and make sure the internet connection is stable for playing. Physiotherapists and general practitioners who best would prescribe VR currently lack the supporting logistics to provide patients with these education sessions and technical support.

For that, ideally, VR would be embedded within a larger virtual care platform. These platforms already provide telemedicine and have the infrastructure to add VR to their service lines. ${ }^{24}$ Such services are expected to exponentially increase during and in the aftermath of this pandemic. Healthcare practitioners could involve virtual care platforms to lend or lease VR headsets to patients for use at home, as purchasing a headset is ideally not the responsibility of a patient and is at the long run not a sustainable business model. Ideally, costs would be covered by general health insurance, making VR equally available for anyone. First steps have been made recently when insurance companies decided to reimburse rehabilitation for COVID-19 patients, which can include VR treatment. Virtual care platforms could consequently facilitate the need for education to teach patients to use VR headsets. Education sessions could be provided remotely to patients personalised to their level of experience with computer technologies. If needed, these sessions could be supported face-to-face by a skilled family member. On-demand support should be constantly available for answering questions and solving technological artefacts. When virtual care platforms fulfil criteria of privacy and security, physiotherapists can safely monitor progress of patients and adjust and communicate treatment plans accordingly.

The authors have set up a scientific study for assessing VR for rehabilitation from COVID-19 (study identification name and number: COVRehab, NCT04505761). All patients after COVID-19 who need physical therapy are eligible for this study, regardless of whether they have or have not been admitted to the hospital and/or ICU. This choice was made based on early experiences with serious postinfectious complaints of patients who did not have to be treated in the hospital. ${ }^{25}$ Study results will facilitate the collection of critical data regarding implementation of VR for rehabilitation.

\section{CONCLUSION}

The COVID-19 crisis has generated a need to expand telehealth technologies. We believe that VR therapy offers an opportunity for recovering COVID-19 patients and care providers to partake in efficient home-based and individualised rehabilitation, monitored at a distance. Several barriers for implementation will need to be overcome, including designing an appropriate, safe, easy-to-use and cost-effective VR toolkit, organising home-based VR therapy and support at a distance, and reimbursement. Embedding VR in virtual care platforms would assist in overcoming these barriers and stimulating the spread of VR therapy, both for post-COVID-19 patients in the present and possibly for other patients with similar rehabilitation needs in the future.

Contributors All authors participated in the conceptualisation of the article. MS wrote the first draft, BS and HvG reviewed and provided comments. All authors reviewed, commented on and approved the final draft.

Funding The authors have not declared a specific grant for this research from any funding agency in the public, commercial or not-for-profit sectors.

Competing interests None declared.

Patient consent for publication Not declared.

Ethics approval No ethical approval was obtained as no study participants were involved.

Provenance and peer review Not commissioned; externally peer reviewed.

Data availability statement No additional data are available.

Open access This is an open access article distributed in accordance with the Creative Commons Attribution Non Commercial (CC BY-NC 4.0) license, which permits others to distribute, remix, adapt, build upon this work non-commercially, and license their derivative works on different terms, provided the original work is properly cited, appropriate credit is given, any changes made indicated, and the use is non-commercial. See: http://creativecommons.org/licenses/by-nc/4.0/.

\section{ORCID iDs}

Merlijn Smits http://orcid.org/0000-0002-2292-6950

J Bart Staal http://orcid.org/0000-0002-0083-6380

Harry van Goor http://orcid.org/0000-0003-0323-4876

\section{REFERENCES}

1 Biehl M, Sese D. Post-intensive care syndrome and COVID-19 implications post pandemic. Cleve Clin J Med 2020.

2 Inoue S, Hatakeyama J, Kondo Y, et al. Post-intensive care syndrome: its pathophysiology, prevention, and future directions. Acute Med Surg 2019;6:233-46.

3 Thornton J. COVID-19: the challenge of patient rehabilitation after intensive care. BMJ 2020;369.

4 Barker-Davies RM, Sullivan O, Senaratne KPP, et al. The Stanford Hall consensus statement for post-COVID-19 rehabilitation. Br J Sports Med 2020; bjsports-2020-102596.

5 Simpson R, Robinson L. Rehabilitation following critical illness in people with COVID-19 infection. Am J Phys Med Rehabil 2020;1.

6 Phillips M, Turner-Stokes L, Wade D, et al. Rehabilitation in the wake of COVID-19 - a phoenix from the ashes. Br Rehabil Med 2020. Available https://www.bsrm.org.uk/downloads/covid-19bsrmissue1-published27-4-2020.pdf

7 Davidson JE, Harvey MA. Patient and family post-intensive care syndrome. AACN Adv Crit Care 2016;27:184-6.

8 Bevilacqua R, Maranesi E, Riccardi GR, et al. Non-immersive virtual reality for rehabilitation of the older people: a systematic review into efficacy and effectiveness. J Clin Medi 2019;8:1882.

9 Lin H-T, Li Y-I, Hu W-P, et al. A scoping review of the efficacy of virtual reality and exergaming on patients of musculoskeletal system disorder. $J$ Clin Medi 2019;8:791.

10 Dorsey ER, Topol EJ. State of telehealth. N Engl J Med 2016;375:154-61.

11 De Graaf J, Behandelprogramma BM COVID-19 post-IC de Hoogstraat. Online, 2020.

12 Yang $\mathrm{F}$, Liu N, Hu J, et al. Pulmonary rehabilitation guidelines in the principle of 4S for patients infected with 2019 novel coronavirus (2019-nCoV). Chin J Tuberculosis Respir Dis 2020;43:180-2.

13 Darekar A, McFadyen BJ, Lamontagne A, et al. Efficacy of virtual reality-based intervention on balance and mobility disorders post-stroke: a scoping review. J NeuroEng Rehabil 2015;12:46.

14 Wu J, Loprinzi PD, Ren Z. The rehabilitative effects of virtual reality games on balance performance among children with cerebral palsy: a 
meta-analysis of randomized controlled trials. Int J Environ Res Public Health 2019;16:4161.

15 Pourmand A, Davis S, Marchak A, et al. Virtual reality as a clinical tool for pain management. Curr Pain Headache Rep 2018;22:53.

16 Li J, Theng Y-L FS. Game-based digital interventions for depression therapy: a systematic review and meta-analysis. Cyberpsychol Behav Soc Netw 2014;17:519-27.

17 Meyerbröker K, Emmelkamp PM. Virtual reality exposure therapy in anxiety disorders: a systematic review of process-and-outcome studies. Depress Anxiety 2010;27:933-44.

18 Gonçalves R, Pedrozo AL, Coutinho ESF, et al. Efficacy of virtual reality exposure therapy in the treatment of PTSD: a systematic review. PloS One 2012;7:12.

19 Honarmand K, Lalli RS, Priestap F, et al. Natural history of cognitive impairment in critical illness survivors: a systematic review. Am J Respir Crit Care Med. 2020.

20 Zhao J, Yao L, Wang C, et al. The effects of cognitive intervention on cognitive impairments after intensive care unit admission. Neuropsychol Rehabil 2017;27:301-17.
21 Cho DR, Lee SH. Effects of virtual reality immersive training with computerized cognitive training on cognitive function and activities of daily living performance in patients with acute stage stroke: a preliminary randomized controlled trial. Medicine 2019;98: e14752.

22 Warwick U. Physiotherapy could be done at home using virtual reality online 2020 Available https://medicalxpress.com/news/2020-02physiotherapy-home-virtual-reality.html?utm_content= 132844014\&utm_medium=social\&utm_source=linkedin\&hss_chan nel=lis-kQX_GK_W7C

23 Torous J, Wykes T. Opportunities from the coronavirus disease 2019 pandemic for transforming psychiatric care with telehealth. JAMA Psychiatry 2020.

24 Irgens I, Rekand T, Arora M, et al. Telehealth for people with spinal cord injury: a narrative review. Spinal Cord 2018;56:643-55.

25 Koninklijk Nederlands Genootschap voor Fysiotherapie. KNGF position statement - physiotherapy recommendations in patients with COVID-19. Amersfoort: Royal Dutch Society for Physical Therapy (KNGF), 2020 